\title{
De los genes a la atención clínica -oncología bucal-
}

Since genes to clinical care-buccal oncology-

\author{
Brunotto M \\ Universidad Nacional de Córdoba, Facultad de Odontología, Departamento de Biología Bucal, \\ Instituto de Investigaciones en Ciencias de la Salud de Córdoba (INICSA-CONICET,) Argentina
}

\begin{abstract}
The prevention of cancer is one of the best strategies at the Public Health level since it is a low cost method and highly effective over time. Early detection allows intervention in the early stages of the disease, this being a moment with high potential to achieve healing. The evaluation of diagnostic indicators, such as symptoms and genetic tests, allows increasing the sensitivity and specificity of the determination of the presence / absence of a malignant pathology. A description of profiles of biomarkers in relation to changes in the profiles of these markers at different times allows us to assess, for example, the progression towards malignization of patients with premalignant lesions.
\end{abstract}

KEYWORDS: genetic, oncology, buccal cavity

\section{Resumen}

La prevención del cáncer es una de las mejores estrategias a nivel de Salud Pública ya que es un método de bajo costo y alta efectividad en el tiempo. La detección precoz permite intervenir en los estadios tempranos de la enfermedad, siendo este un momento con alto potencial para lograr la curación. La evaluación de indicadores diagnósticos, tales como síntomas y pruebas genéticas, permite incrementar la sensibilidad y especificidad de la determinación de la presencia/ausencia de una patología maligna. Una descripción de perfiles de biomarcadores en relación con cambios en los perfiles de esos marcadores en distintos tiempos permite valorar por ejemplo la progresión hacia la malignización de pacientes con lesiones premalignas.

PALABRAS CLAVE: genética, oncología, cavidad bucal

\section{Introducción}

En América se está observando una creciente prevalencia de enfermedades crónicodegenerativas, sumadas a las enfermedades infecto-contagiosas ya existentes en la región ${ }^{1}$. Sumado a estos hechos, la ciencia y el desarrollo tecnológico han avanzado rápidamente $\mathrm{e}$ incorporan los nuevos descubrimientos y cambios de paradigmas en la prevención-atención en la medicina. Ejemplo de esto son las bio-drogas, robótica, nano-dispositivos, vacunas terapéuticas, tratamientos individualizados. La genómica médica está dirigida a una medicina predictiva, personalizada y preventiva. Sin embargo, esto genera una desigualdad en el acceso a la obtención de esta nueva tecnología por los sectores socio-económicamente bajos. Por lo cual se hace necesario en este contexto que los sistemas de salud utilicen de modo adecuado la información y la comunicación para la construcción de una conciencia crítica y política que llegue a toda la población mejorando la calidad de vida de la misma ${ }^{1}$.

La prevención del cáncer es una de las mejores estrategias a nivel de Salud Pública ya que es un método de bajo costo y alta efectividad en el tiempo. La detección precoz permite intervenir en los estadios tempranos de la enfermedad, siendo este un momento con alto potencial para lograr la curación.

La evaluación de indicadores diagnósticos, tales como síntomas y pruebas genéticas, permite 
incrementar la sensibilidad y especificidad de la determinación de la presencia/ausencia de una patología maligna ${ }^{2}$. Una descripción de perfiles de biomarcadores en relación con cambios en los perfiles de esos marcadores en distintos tiempos permite valorar por ejemplo la progresión hacia la malignización de pacientes con lesiones premalignas $^{3}$.

Desde hace varias décadas se intenta avanzar en reconocer marcadores genéticos pronósticos de riesgo de cáncer y/o de progresión tumoral a fin de mejorar las acciones de prevención, detección temprana y tratamiento.Las investigaciones genéticas de enfermedades complejas se han incrementado considerablemente, especialmente los relacionados a estudios de genoma completo (GWAS, del inglés Genome-Wide Association Studies). Este tipo de estudios ha generado una valiosa herramienta para la identificación de nuevos polimorfismos o mutaciones genéticas involucradas en la susceptibilidad al cáncer. Los GWAS investigan el conjunto del genoma mediante la exploración de las asociaciones entre un gran número de polimorfismos. Hasta la fecha, Se han publicado más de 500 GWAS de cáncer, incluyendo cáncer de mama, próstata, colorrectal y pulmón, identificados en diferentes poblaciones, permitiendo comprender mejor los mecanismos genéticos involucrados en el desarrollo de muchos tipos de cáncer. La aplicación clínica de los estudios GWAS proporciona oportunidades para el descubrimiento de fármacos como para la prevención del cáncer, aunque su implementación en salud pública se ha visto obstaculizada por la falta de recursos disponibles, así como infraestructuras complejas y personal con experiencia en el área ${ }^{4}$.

Sin embargo es una temática importante de continuar en investigación básica para su aplicación en la clínica.

El autor declara que no existen conflictos potenciales de interés con respecto a la autoría y / o publicación de este artículo.

The author declares no potential conflicts of interest with respect to the authorship and/or publication of this article.

\section{Referencias}

1. Giovanella L,Feo O, Faria M, Tobar S (orgs.).Sistemas de salud en Suramérica: desafíos para launiversalidad la integralidad y la equidad / InstitutoSuramericano de Gobierno en Salud; Rio de Janeiro: ISAGS, 2012. Disponible en:http://www.slideshare.net/isagsunasur/sistemas-desalud-en-suramerica.

2. Lingen MW, Pinto A, Mendes RA, Franchini R, Czerninski R, Tilakaratne WM, et al. Genetics/epigenetics of oral premalignancy: current status and future research. Oral Dis.2011; 17:7-22

3. Zarate AM, BrezzoMM, Secchi DG, Barra JL, BrunottoM. Malignancy Risk Models for Oral Lesions. Med Oral Patol Oral Cir Bucal. 2013. doi:10.4317/medoral.18374.

4. Vukovic V, Stojanovic J, Vecchioni A, Pastorino R, Boccia S. Systematic Review and Meta-analysis of SNPs from Genome-Wide Association Studies of Head and Neck Cancer. Otolaryngol Head Neck Surg. 2018; 159(4):615-624. doi: 10.1177/0194599818792262

Correspondencia a/Corresponding to: Dr. Mabel Brunotto Universidad Nacional de Córdoba, Facultad de Odontología, Departamento de Biología Bucal, Argentina Instituto de Investigaciones en Ciencias de la Salud de Córdoba (INICSA-CONICET)

Pabellón Argentina, Haya de la Torre $s / n$ Correo electrónico/E-mail: brunottomabel@gmail.com, mabel.brunotto@unc.edu.ar 
